\title{
Determinants of virological outcome and adverse events in African children treated with paediatric nevirapine fixed-dose-combination tablets
}

\author{
Andrzej Bienczaka, Paolo Denti ${ }^{\mathrm{a}}$, Adrian Cook ${ }^{\mathrm{b}}$, Lubbe Wiesner ${ }^{\mathrm{a}}$, \\ Veronica Mulenga ${ }^{c}$, Cissy Kityo ${ }^{d}$, Addy Kekitiinwa ${ }^{\text {e,f }}$, Diana M. Gibb ${ }^{b}$, \\ David Burgerg, Ann S. Walker ${ }^{\mathrm{b}, *}$ and Helen Mcllleron ${ }^{\mathrm{a}, *}$
}

\begin{abstract}
Background: Nevirapine is the only nonnucleoside reverse transcriptase inhibitor currently available as a paediatric fixed-dose-combination tablet and is widely used in African children. Nonetheless, the number of investigations into pharmacokinetic determinants of virological suppression in African children is limited, and the predictive power of the current therapeutic range was never evaluated in this population, thereby limiting treatment optimization.
\end{abstract}

Methods: We analysed data from 322 African children (aged 0.3-13 years) treated with nevirapine, lamivudine, and either abacavir, stavudine, or zidovudine, and followed up to 144 weeks. Nevirapine trough concentration $\left(C_{\mathrm{min}}\right)$ and other factors were tested for associations with viral load more than $100 \mathrm{copies} / \mathrm{ml}$ and transaminase increases more than grade 1 using proportional hazard and logistic models in 219 initially antiretroviral treatment (ART)-naive children.

Results: Pre-ART viral load, adherence, and nevirapine $C_{\min }$ were associated with viral load nonsuppression [hazard ratio $=2.08$ (95\% confidence interval $(\mathrm{Cl}): 1.50-2.90$, $P<0.001)$ for 10 -fold higher pre-ART viral load, hazard ratio $=0.78(95 \% \mathrm{Cl}: 0.68-$ $0.90, P<0.001)$ for $10 \%$ improvement in adherence, and hazard ratio $=0.94(95 \% \mathrm{Cl}$ : $0.90-0.99, P=0.014)$ for a $1 \mathrm{mg} / \mathrm{l}$ increase in nevirapine $\left.C_{\min }\right]$. There were additional effects of pre-ART $\mathrm{CD}^{+}$cell percentage and clinical site. The risk of virological nonsuppression decreased with increasing nevirapine $C_{\min }$, and there was no clear $C_{\min }$ threshold predictive of virological nonsuppression. Transient transaminase elevations more than grade 1 were associated with high $C_{\min }(>12.4 \mathrm{mg} / \mathrm{l})$, hazard ratio $=5.18(95 \% \mathrm{Cl} 1.95-13.80, P<0.001)$.

Conclusion: Treatment initiation at lower pre-ART viral load and higher pre-ART CD4 ${ }^{+}$ cell percentage, increased adherence, and maintaining average $C_{\min }$ higher than current target could improve virological suppression of African children treated with nevirapine without increasing toxicity.

Copyright (C) 2017 The Author(s). Published by Wolters Kluwer Health, Inc.

AIDS 2017, 31:905-915

Keywords: antiretroviral treatment efficacy, CHAPAS-3, nevirapine pharmacokinetics/pharmacodynamics, paediatric HIV

\footnotetext{
${ }^{a}$ Division of Clinical Pharmacology, Department of Medicine, University of Cape Town, Cape Town, South Africa, ${ }^{b}$ MRC Clinical Trials Unit at University College London, London, United Kingdom, ' Department of Paediatrics and Child Health, University Teaching Hospital, Lusaka, Zambia, 'Joint Clinical Research Centre, 'Baylor College of Medicine Bristol Myers Squibb Children's Clinical Centre of Excellence, Kampala, Uganda, ' Gulu Regional Centre of Excellence, Gulu, Uganda, and ${ }^{\mathrm{g}}$ Department of Pharmacy, Radboud University Medical Centre, Nijmegen, The Netherlands.

Correspondence to Helen Mcllleron, PhD, Groote Schuur Hospital, Observatory, K45 Old Main Building, Cape Town 7925, South Africa.
}

Tel: +27 21406 6292; fax: +27 21448 1989; e-mail: helen.mcilleron@uct.ac.za

* Ann S. Walker and Helen Mcllleron contributed equally to the article.

Received: 14 October 2016; revised: 5 December 2016; accepted: 5 December 2016.

DOI:10.1097/QAD.0000000000001376

ISSN 0269-9370 Copyright $\subset 2017$ The Author(s). Published by Wolters Kluwer Health, Inc. This is an open access article distributed under the terms of the Creative Commons Attribution-Non Commercial License 4.0 (CCBY-NC), where it is permissible to download, share, remix, transform, and buildup the work provided it is properly cited. The work cannot be used commercially without permission from the journal. 


\section{Introduction}

Fixed-dose combination (FDC) formulations have considerably improved access to antiretroviral treatment (ART) through decreased cost and improved feasibility, especially in sub-Saharan Africa [1]. Currently available paediatric-dispersible FDCs are limited to combinations of a nonnucleoside reverse transcriptase inhibitor (NNRTI), nevirapine, with two nucleoside-reverse transcriptase inhibitors (NRTIs), and are widely used in children in low-income countries [2].

Nevirapine pharmacokinetics exhibits high variability, attributed in part to single nucleotide polymorphisms (SNPs) of cytochrome P450 2B6 (CYP2B6), which encode an important metabolic pathway for this drug [35]. In adults, low nevirapine concentrations have been associated with increased risk of virological failure [6-8] and high exposures with increased risk of skin rashes [9$11]$ and hepatotoxicity [7,12]. Based on the concentration-response relationship, a therapeutic range of 3$8 \mathrm{mg} / 1$ has been suggested for nevirapine therapeutic drug monitoring [13]. However, several studies failed to confirm these associations $[4,14,15]$, and low incidences of nevirapine-related adverse events have been reported in low-income settings [16] and in African children $[17,18]$. Despite widespread use, few studies have investigated the pharmacokinetic determinants of efficacy of nevirapine-based regimens in children. The predictive power of the suggested targets has also never been thoroughly investigated in black Africans or in children. Whether these pharmacokinetic targets should be universally applied across populations was recently questioned [4].

Our aim was therefore to investigate associations between nevirapine trough concentrations $\left(C_{\mathrm{min}}\right)$ and long-term virological outcomes and adverse events in African children and establish if any other factors predict treatment outcome after adjusting for drug exposures, allowing treatment optimization.

\section{Methods}

\section{Population and study design}

The Children with HIV in Africa - Pharmacokinetics and Adherence/Acceptability of Simple Antiretroviral Regimens (CHAPAS-3) trial enrolled HIV-infected ARTnaive and ART-experienced $(>2$ years ART with viral load $<50$ copies $/ \mathrm{ml}$ at screening) children aged $0.3-13$ years from four sites in Uganda and Zambia [19], treated following WHO 2010 guidelines [20] with an NNRTI (nevirapine or efavirenz) and two NRTIs (lamivudine and randomized abacavir, stavudine, or zidovudine). Nevirapine was coformulated with companion NRTIs in paediatric FDCs provided by Cipla (Mumbai,
Maharashtra, India) [19]. Children on nevirapine were switched to efavirenz-based ART if aged more than 3 years and diagnosed with tuberculosis (TB) or experienced nevirapine-related adverse events, or to boosted protease inhibitor-based ART if less than 3 years with these events or for clinical or immunological failure (or if efavirenz-intolerant). Samples for pharmacokinetics analysis (described previously) [5] were taken at weeks 6, 36 , and every 24 weeks thereafter. Viral load was assayed retrospectively on stored plasma taken at enrolment and weeks 48, 96, 132, or 144 .

\section{Statistical analysis}

A previously developed model describing the steady-state population pharmacokinetics of nevirapine [5] was used to derive empirical Bayesian estimates for each child at each pharmacokinetic visit for clearance, $C_{\min }$ (evening trough concentration), $C_{\max }$ (maximum concentration), and $\mathrm{AUC}_{0-24}$ (area under the curve). Due to diurnal variability in clearance [5], this analysis included measurements relating to daytime exposures only.

The primary efficacy outcome was viral load more than 100 copies/ $\mathrm{ml}$ (the limit of detection as many samples had to be diluted due to low volumes). For descriptive analysis, response was categorized as suppressed $(<100$ copies $/ \mathrm{ml}$ within 48 weeks of treatment initiation, maintained throughout follow-up), slow suppression $(<100$ copies $/ \mathrm{ml}$ achieved after 48 weeks but maintained throughout subsequent follow-up), rebounded $(<100$ copies/ml within 48 weeks but viral load $>100$ copies $/ \mathrm{ml}$ at single or multiple visits thereafter), and never suppressed (viral load never $<100$ copies/ml). ARTexperienced children (all viral load $<50$ copies $/ \mathrm{ml}$ at enrolment) were analysed separately from ART-naive children initiating treatment at enrolment. As multiple pharmacokinetic exposures were available for each child, geometric means of pharmacokinetics parameters across follow-up within each individual, and interindividual variability (expressed as coefficient of variation [21]) for $C_{\min }$ and $\mathrm{AUC}_{0-24}$ were compared between groups using Kruskal-Wallis and rank-sum tests. Categorical factors were compared between groups using Fisher's exact test.

The effect of nevirapine $C_{\min }$ on the risk of virological nonsuppression $(>100$ copies $/ \mathrm{ml})$ in the subset of ARTnaive children only was estimated using Cox proportional hazards regression models (Andersen-Gill repeated outcomes framework) with Efron approximation in $\mathrm{R}$ (survival package) [22-25]. Viral loads were matched with the model estimated $C_{\min }$ from the closest sampling visit preceding each viral load. Each time interval ran from the preceding to the current viral load (classified as suppressed vs nonsuppressed 'event'), and the matched $C_{\min }$ was applied to the whole time interval. Nonlinearity in the effect of $C_{\min }$ was explored visually using smoothed splines and tested using fractional polynomials (Stata $\mathrm{mfp}$ cox; Stata Statistical Software: Release 14. StataCorp. LP, 
College Station, Texas, USA) [26]. As Cox regression does not provide estimates of the absolute probability of suppression, we estimated this using a mixed-effects repeated measures logistic model (Stata mfp logistic; StataCorp. LP) [26]. The best-fitting dichotomous threshold for nevirapine $C_{\min }$ in the Cox model was identified by profile likelihood, as described previously for efavirenz [27]. Following the same method, we conducted simulations introducing unexplained residual variability on predicted concentrations from the population pharmacokinetics model (additive error $0.32 \mathrm{mg} / 1$, proportional error 5.26\%) [5] to derive 95\% confidence intervals (CIs) for this threshold (2.5th and 97.5th percentile of most predictive cut-offs from 500 simulations). The sensitivity, specificity, accuracy, and positive and negative predictive values of the identified threshold for viral load suppression were compared with those of the 10th, 25th, and 50th percentiles of estimated nevirapine $C_{\min }$ in this study and cut-offs proposed in the literature $[6,7,13]$.

Finally, we used backwards elimination (exit $P=0.05$, retaining all levels of categorical factors where $P<0.05$ ) to consider the additional independent effects on nonsuppression of factors with associations $(P<0.2)$ in univariate models. Categorical covariates included NRTI-backbone (abacavir, zidovudine, or stavudine), sex, clinical site, exposure to ART in children and/or mothers in prevention of mother-to-child transmission [pMTCT (regimens listed in footnote to Table 1)], metabolizer status (MET) based on CYP2B6 516G $>$ T $\mid 983 \mathrm{~T}>\mathrm{C}$ SNP (extensive metabolizers 516GG|983TT; intermediate metabolizers 516GG|983TC or 516GT|983TT; slow metabolizers 516TT|983TT or 516GT|983TC; and ultraslow metabolizers - 516GG|983CC) [5], mother as primary carer, and self-reported missing of any ART doses in previous 4 weeks. Continuous variables included baseline (preART) viral load (bVL) and $\mathrm{CD}^{+}$cell percentage (bCD4 ${ }^{+}$cell percentage), truncated at $50 \%$ to avoid undue influence of outliers), current age, weight-for-age $Z$-score (WAZ) [28], height-for-age $Z$-score (HAZ) [28], and adherence [percentage of doses taken based on Medication Event Monitoring Systems (MEMS)-cap container openings in the interval between previous and current viral load (truncated to a lower limit of 0.5)]. The only factor with incomplete information was adherence; when no data were available for current interval, the preceding interval's value was carried forward. If no prior MEMS data were available $(n=21)$, we imputed the median value for all ART-naive individuals. Nonlinear effects in continuous variables were explored as described above for $C_{\min }$. Interactions between factors included in the final model were investigated and included if $P$ was less than 0.05 .

\section{Adverse events}

Adverse events considered to be nevirapine-related were hypersensitivity reactions [HSR, including Stevens-
Johnson Syndrome (SJS)], raised liver enzymes [aspartate or alanine transaminase (AST or ALT) $>$ grade 2 , i.e. $>5 \times$ upper limit of normal (ULN)], and acute hepatitis. The characteristics of children developing adverse events were compared with others using Fisher's exact or rank-sum tests. AST and ALT were measured at enrolment and weeks $6,12,24$, and 24-weekly throughout the study and were matched with nevirapine $C_{\min }$ as described for viral load. The association between nevirapine $C_{\min }$ (and all covariates above and pre-ART more than grade 1 transaminase elevation) and the risk of developing more than grade 1 AST or ALT, that is, more than $2.5 \times$ ULN (composite endpoint), was estimated in the ART-naive group as for virological nonsuppression. In addition, in the same group, the change from baseline in transaminase levels at weeks 6, 48, and 96 was compared using Wilcoxon signed rank test separately for children with $C_{\min }$ below and above the threshold identified most predictive of transaminase more than 1 grade elevations by likelihood profiling as explained above. Probabilities of adverse events were similarly estimated using mixedeffects logistic regression.

\section{Results}

\section{Patient characteristics}

Of 478 children in CHAPAS-3, 338 received nevirapine (99 ART-experienced) combined with a two-NRTI backbone, and contributed 3340 pharmacokinetics samples (1566 dosing intervals, 1-6 per individual) and 718 viral loads after enrolment (1-3 per individual). Sixteen individuals (all ART-naive) changed ART: nine to efavirenz (when TB was diagnosed) and seven to protease-inhibitors [three for adverse events, four for clinical failure (two in year 2 and two in year 3)]. The demographic characteristics and model-estimated pharmacokinetics parameters for children included in this analysis are shown in Table 1 by virological response group.

Amongst ART-naive children, 151 (68\%) achieved and maintained viral load less than 100 copies/ml, 125 (56\%) by week 48. Those who took longer to suppress had almost three times higher pre-ART viral load and lower $\mathrm{CD}^{+}{ }^{+}$cell counts. Amongst ART-naive participants who suppressed by week 48, 27 rebounded, and the majority of these children resuppressed during follow-up. Pre$\mathrm{ART} \mathrm{CD}^{+}{ }^{+}$cell percentage in these rebounders was lower than other groups, and median pre-ART viral load between that for the suppressed group and for those taking longer to suppress or never suppressed. The remaining 45 ART-naive children (20\%) never suppressed viral load to less than 100 copies/ml, but only four showed clinical evidence of treatment failure. Individuals who never suppressed had significantly higher pre-ART viral load than children who suppressed by week 48 , 


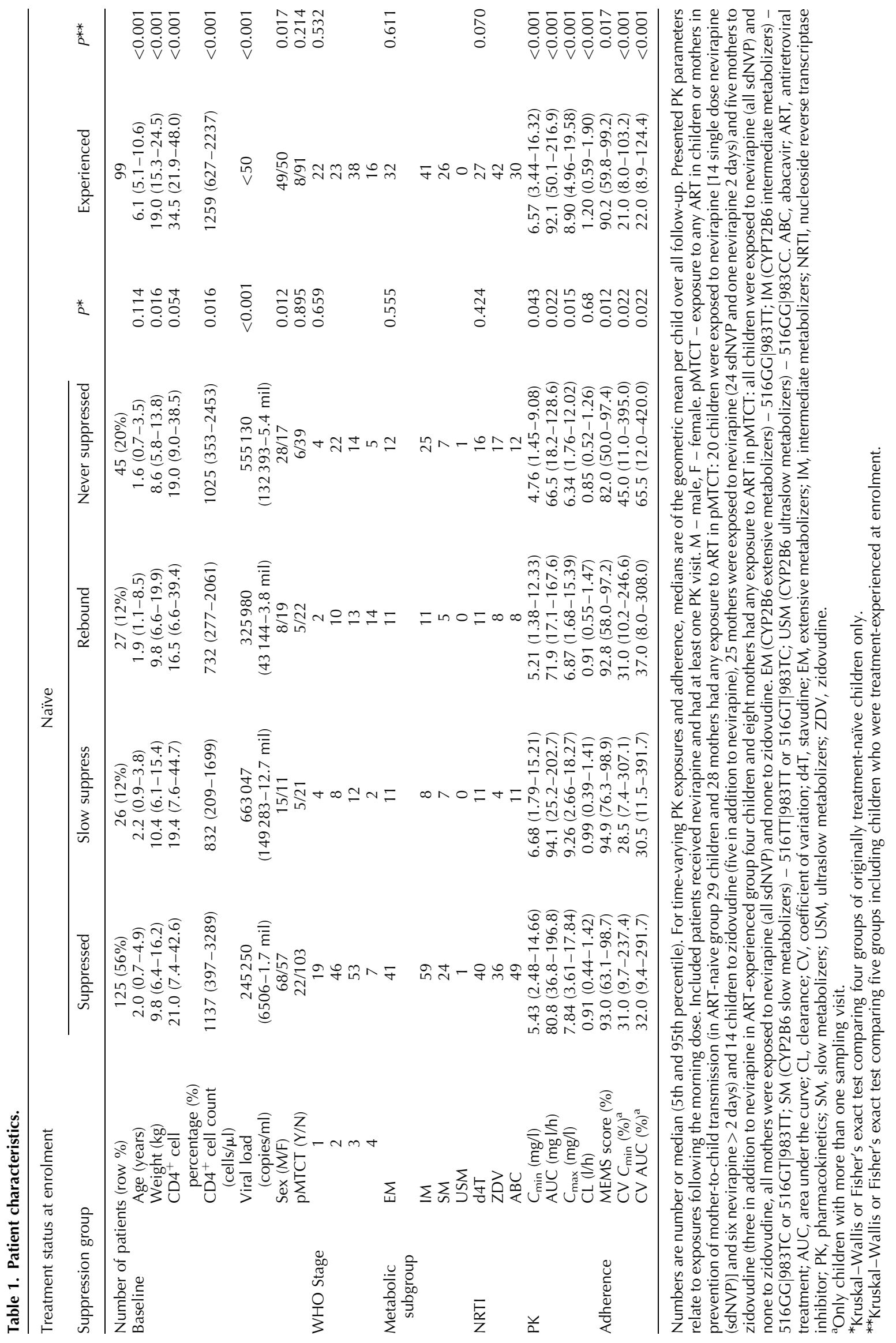


lower adherence than the other ART-naive children $(82$ vs $93 \%$, rank-sum $P=0.005)$ and lower nevirapine pharmacokinetics exposures $(P<0.05)$ with higher levels of intraindividual variability $(P=0.02)$, possibly indicating erratic adherence patterns.

ART-experienced children were much older, with viral load less than 50 copies $/ \mathrm{ml}$ and higher $\mathrm{CD}^{+}$cell percentage at enrolment. The average $C_{\min }$ and AUC in this group was also higher than most ART-naive children $(P<0.001)$. Despite comparable MEMS-adherence scores, ART-experienced children had significantly lower intraindividual variability in nevirapine pharmacokinetics measures than ART-naive children $(P<0.001)$, which might suggest more consistent adherence. Virological outcomes remained excellent: 88 (89\%) remained suppressed less than 100 copies $/ \mathrm{ml}$ throughout the study, $10(10 \%)$ had a virological rebound, and only one child had all viral load measurements more than 100 copies $/ \mathrm{ml}$.

\section{Concentration-response relationship}

Cox repeated failures regression on 437 matched pharmacokinetics viral load measurements in 219 ART-naive individuals (Table 2) showed that the hazard of nonsuppression decreased by $7 \%$ for every $1 \mathrm{mg} / 1$ increase in nevirapine $C_{\min }(95 \% \mathrm{CI}: 2-12 \%)$. The estimated probability of nonsuppression declined from $26 \%$ for a nevirapine $C_{\min }$ of $3 \mathrm{mg} / 1$ to 18,12 , and $9 \%$ for $C_{\min }$ values of 8,12 , and $16 \mathrm{mg} / 1$, respectively, using the mixed-effects repeated measures logistic model (Fig. 1a). Likelihood profiling identified a nevirapine $C_{\min }$ of $10.2 \mathrm{mg} / 1$ (95\% CI 7.9-11.8) as most predictive of decreased risk of virological nonsuppression (Supplement Fig. S1a, http://links.lww.com/QAD/B33). Despite the markedly decreased probability of nonsuppression with
$C_{\min }$ above this threshold and improved specificity and positive predictive value, in comparison with the other $C_{\min }$ cut-offs, the identified threshold had inferior sensitivity, accuracy, and negative predictive power (Table 3).

\section{Predictors of virological nonsuppression}

Nevirapine $C_{\min }$, clinical site, age, WAZ, HAZ, adherence, $\mathrm{bCD} 4^{+}$cell percentage, and $\mathrm{bVL}$ were all associated with viral load more than 100 copies $/ \mathrm{ml}$ in univariate analyses $(P<0.2)$. However, only $C_{\text {min }}$, clinical site, adherence, $\mathrm{bCD} 4^{+}$cell percentage, and bVL were independent predictors $(P<0.05)$. After adjusting for these factors, the effect of $C_{\min }$ dropped slightly from 7 to $6 \%(95 \%$ CI $1-10 \%)$ (Table 2). The strongest predictors were adherence and bVL. Every 10\% increase is MEMS score was associated with a $22 \%$ reduction (95\% CI 10-32\%), and a 10-fold higher bVL was associated with a 2.08 -fold increase $(95 \%$ CI $1.50-$ $2.90)$ in the risk of nonsuppression. Furthermore, for every $10 \%$ increase in bCD $4^{+}$cell percentage, the risk of viral nonsuppression was 29\% (95\% CI 5-46\%) lower. The hazard of nonsuppression was significantly greater at two of the three sites in Uganda, even after adjusting for other significant effects (characteristics by site in Supplement Table S1, http://links.lww.com/QAD/ B33). No significant interactions were detected between predictors in the final model; in particular, there was no evidence that associations between nevirapine exposure and nonsuppression varied by centre (Site $1-$ ref, Site 2 $P=0.23$, Site $3-0.51$, Site $4-P=0.09$ ).

\section{Adverse events}

Skin reactions were rare (four grade-2 HSR, one grade-3 HSR, and one grade-4 SJS). All occurred in ART-naive

Table 2. Univariate and multivariate predictors of virological suppression on nevirapine.

\begin{tabular}{|c|c|c|c|c|}
\hline \multirow[b]{2}{*}{ Factor } & \multicolumn{2}{|c|}{ Univariate $^{\mathrm{a}}$} & \multicolumn{2}{|c|}{ Final multivariate model ${ }^{\mathrm{b}}$} \\
\hline & $\operatorname{HR}(95 \% \mathrm{Cl})$ & $P$ & $\operatorname{HR}(95 \% \mathrm{Cl})$ & $P$ \\
\hline$C_{\min }$ (per $1 \mathrm{mg} / \mathrm{l}$ higher) & $0.93(0.88-0.98)$ & 0.004 & $0.94(0.90-0.99)$ & 0.014 \\
\hline \multirow[t]{3}{*}{ Site (1 ref) } & (2) $1.65(0.91-2.99)$ & 0.096 & (2) $1.98(1.01-3.85)$ & 0.045 \\
\hline & (3) $1.02(0.54-1.93)$ & 0.943 & (3) $1.19(0.64-2.23)$ & 0.573 \\
\hline & (4) $1.41(0.72-2.78)$ & 0.315 & (4) $2.58(1.15-5.75)$ & 0.021 \\
\hline Age (per 1 year older) & $0.83(0.71-0.98)$ & 0.034 & & \\
\hline Pre-ART CD $4^{+}$cell percentage (per $10 \%$ higher) & $0.82(0.65-1.03)$ & 0.101 & $0.71(0.54-0.95)$ & 0.019 \\
\hline Pre-ART VL (per 10-fold higher) & $2.26(1.68-3.02)$ & $<0.001$ & $2.08(1.50-2.90)$ & $<0.001$ \\
\hline WAZ (per unit higher) & $0.84(0.69-1.01)$ & 0.069 & & \\
\hline HAZ (per unit higher) & $0.81(0.69-0.95)$ & 0.013 & & \\
\hline MEMS score (per $10 \%$ higher) & $0.87(0.76-0.99)$ & 0.037 & $0.78(0.68-0.90)$ & $<0.001$ \\
\hline \multirow{3}{*}{ WHO clinical stage ( 1 ref $)$} & (2) $1.78(0.81-3.91)$ & 0.154 & & \\
\hline & (3) $1.34(0.61-2.97)$ & 0.466 & & \\
\hline & (4) $2.36(0.87-6.37)$ & 0.090 & & \\
\hline
\end{tabular}

ART, antiretroviral treatment; $\mathrm{Cl}$, confidence interval; $\mathrm{HAZ}$, height-for-age adjusted Z-scores; VL, viral load; WAZ, weight-for-age adjusted Zscores.

a Showing all factors with univariate $P<0.2$, and hence considered for inclusion in the multivariate model.

${ }^{b}$ Based on backwards elimination using exit $P>0.05$. HR, hazard ratio, clinical sites: (1) - University Teaching Hospital, Lusaka, Zambia; (2) Joint Clinical Research Centre, Kampala, Uganda; (3) - Bristol Myers Squibb Children's Clinical Centre of Excellence, Baylor College of Medicine, Kampala, Uganda; (4) - Joint Clinical Research Centre, Gulu, Uganda. 

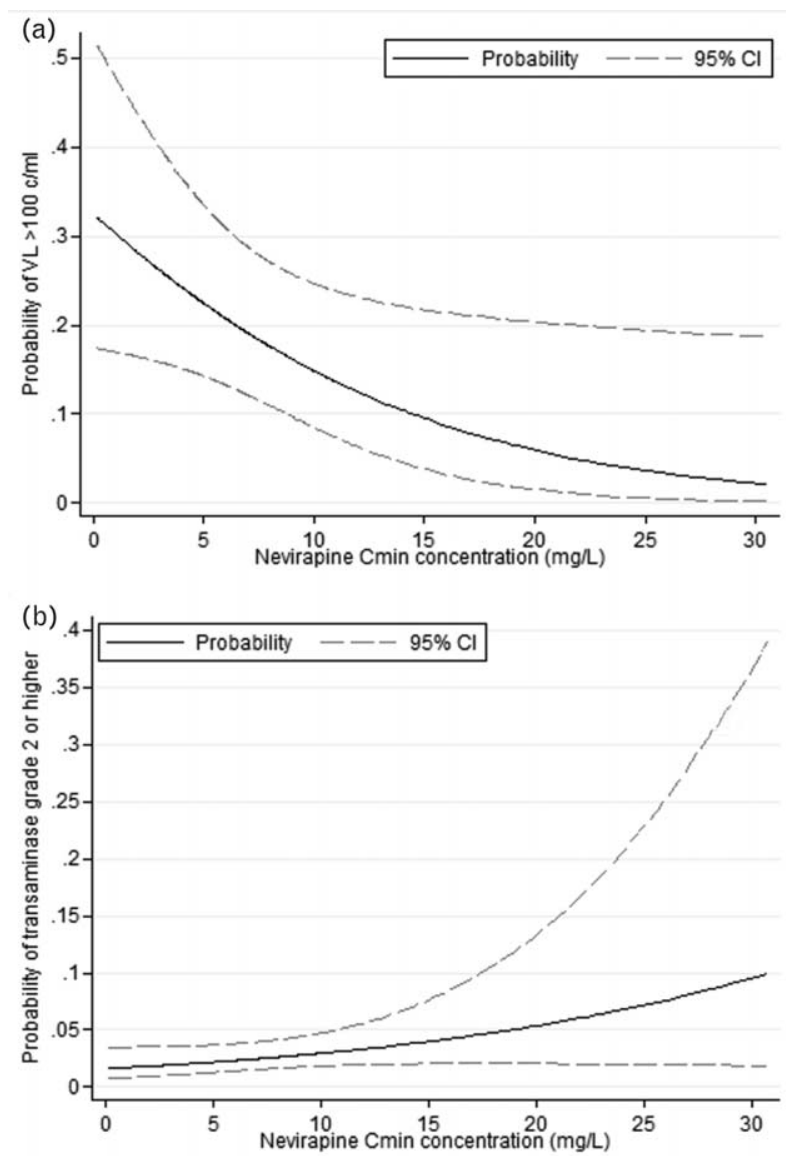

Fig. 1. (a) Probability of nonsuppression (viral load $>\mathbf{1 0 0}$ copies/ml) for nevirapine $C_{\min }$ (b) probability of transaminase grade 2 or higher elevations for nevirapine $C_{\text {min }}$.

patients within 2 weeks of ART initiation, and nevirapine was stopped before pharmacokinetics sampling. The mean pre-ART age and $\mathrm{CD}^{+}$cell percentage were 2.8 years and $22 \%$, respectively, and did not differ significantly from other children (rank-sum $P>0.4$ ); sex was also similar (two boys, four girls; exact $P=0.43$ ) as was CYP2B6-MET (three extensive metabolizers, two intermediate metabolizers, and one slow metabolizers, exact $P=0.87)$.

Transaminase measurements postbaseline were available for 335 children (2273 samples). At enrolment, AST was significantly higher in ART-naive than ART-experienced children with median 43 IU (5th-95th: $26-127$ ) vs 32 IU (22-60), $P$ less than 0.001 , but ALT was similar with median 21 (9-75) vs 23 (13-53), $P=0.14$. Transaminase elevations grade 3 and above were rare (15 in total) and were not associated with any particular characteristics (Supplement Table S2, http://links.lww.com/QAD/ B33), there were no cases of acute hepatitis.

Of 39 more than grade 1 elevations observed in 235 ARTnaïve children, 24 (nine both AST and ALT, six ASTonly,

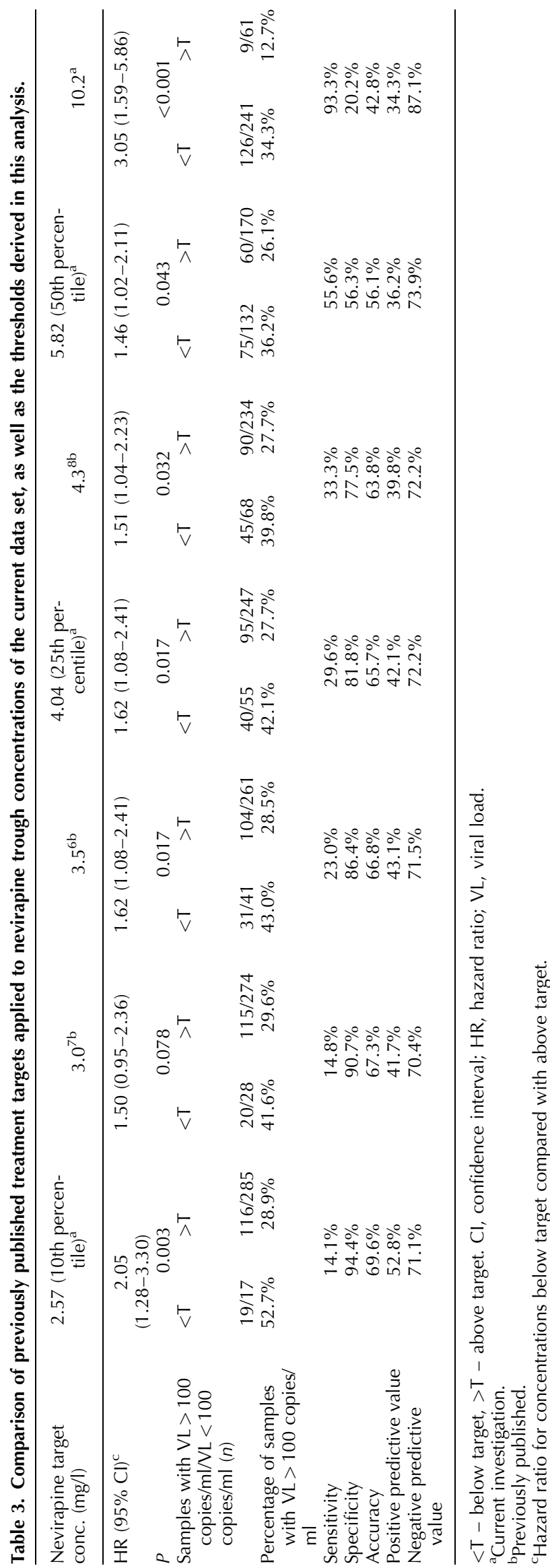

Copyright @ 2017 Wolters Kluwer Health, Inc. All rights reserved. 
and nine ALT only) were matched with nevirapine concentrations and were included in the Cox repeated failures model. The model identified nevirapine $C_{\min }$ [hazard ratio per unit higher (95\% CI) 1.07 (1.01-1.13), $P=0.032]$, but no other factors (including baseline transaminase elevation, sex, age, and WAZ/HAZ) to be univariably associated with increased risk of transaminase grade 2 and above elevations. Likelihood profiling identified $C_{\min }$ cut-off of $12.4 \mathrm{mg} / 1$ (95\% CI $7.7-$ $13.5)$ with hazard ratio ( $95 \% \mathrm{CI}$ ) above vs below the identified threshold of $5.18(1.95-13.80), P$ less than 0.01, (Supplement Fig. S1b, http://links.lww.com/ QAD/B33). All the observed transaminase elevations were transient and none led to change in treatment, and although AST and ALT levels were higher in matched samples with nevirapine $C_{\min }$ more than $12.4 \mathrm{mg} / \mathrm{l}$, at most time points the increase from baseline was not statistically significant (Table 4). The probability of transaminase elevations by nevirapine $C_{\min }$ estimated using mixed-effects repeated measures logistic model are presented in Fig. 1b and $\mathrm{c}$ and remained below $10 \%$ up to $30 \mathrm{mg} / \mathrm{l}$.

\section{Discussion}

We observed that virological nonsuppression in a group of African children treated with nevirapine in combination with two NRTIs was affected by nevirapine $C_{\min }$ and treatment adherence, as well as pre-ART viral load and $\mathrm{CD}^{+}{ }^{+}$cell percentage. Despite confirming a significant concentration-response relationship, we could not identify a meaningful exposure cut-off predictive of virological nonsuppression. Furthermore, other factors independent of systemic exposures were more strongly associated with nonsuppression than nevirapine exposure. Children with lower viral load at ART initiation and better adherence had improved virological outcomes. Adverse events were rare, but high nevirapine $C_{\min }$ was associated with transient grade 1 and above transaminase elevations.

Similar to previous investigations in adults $[6,7,29]$, we confirmed that higher nevirapine concentrations led to superior virological suppression in children. Customarily used efficacy thresholds for nevirapine were derived from distributions of concentrations in adult, predominantly white, patients, even though nevirapine exposures are higher in African populations [10] and children [5,30], bringing into question their universal applicability [4]. We recently proposed an alternative method of selecting an efficacy threshold based on likelihood profiling and successfully used it for efavirenz in a similar population of African children [27]. Interestingly, a similarly clear cutoff could not be derived for nevirapine, in line with findings by Van Leth et al. [31]. The identified $C_{\min }$ threshold of $10.2 \mathrm{mg} / \mathrm{l}$, despite having superior sensitivity 
and negative predictive value, had substantially lower specificity and accuracy than other cut-offs (Table 3 ). In comparison, the threshold identified for efavirenz $\left(C_{\text {min }}\right.$ of $0.65 \mathrm{mg} / \mathrm{l}$ ) was visibly superior to previously suggested and clearly predicted nonsuppression, with only $7 \%$ of samples above it but $37 \%$ below it having viral load more than 100 copies/ml [27]. Nevirapine has lower potency (protein adjusted IC95 of 196.6 vs $54.7 \mathrm{ng} / \mathrm{l}$ ) [32] and shorter half-life than efavirenz, which is the most potent component of NNRTI + two NRTI ART contributing $65 \%$ of its total efficacy [33]. A higher contribution to treatment efficacy of the two accompanying NRTIs may have obscured a clear pharmacokinetics efficacy threshold for nevirapine. The above could also explain why virological outcomes were more strongly related to several other factors than nevirapine exposures in children on nevirapine-based ART.

The effects of pre-ART CD4 ${ }^{+}$cell percentage and viral load on virological outcome have been well documented [34-39]. In CHAPAS-3, ART-naive children on nevirapine with a higher pre-ART viral load either took much longer to achieve viral load less than 100 copies $/ \mathrm{ml}$ or never suppressed, consistent with the increased hazard of virological nonsuppression with higher pre-ART viral load. The pre-ART CD4 ${ }^{+}$cell percentage in ART-naive children who rebounded after achieving initial suppression by week 48 was significantly lower than in other groups, and it was also an independent predictor of virological nonsuppression. Our findings highlight the benefits of treatment initiation in early stages of disease, in children with a low viral load and high $\mathrm{CD}^{+}$cell percentage. The recent START trial [40] in adults confirmed the importance of starting ART early, and supported guidelines recommending initiation of ART regardless of $\mathrm{CD}^{+}$cell count [41].

Our findings emphasize the importance of treatment adherence in achieving and maintaining virological suppression, consistent with other studies in African children $[38,39]$. Children who never achieved viral load less than 100 copies/ml in our study had significantly lower MEMS scores. Adherence also independently predicted virological nonsuppression with risk decreasing by $22 \%$ for every $10 \%$ higher MEMS score. It has been hypothesized that adherence above $95 \%$ is required to achieve and maintain beneficial effects of ART [31,42]. Interestingly, in CHAPAS-3, the median adherence in children taking efavirenz, an NNRTI administered once a day, was higher than for nevirapine (99 vs 91\%) [27]. This could explain why the association between adherence and virological outcome was more significant for nevirapine than efavirenz. Meta-analyses confirm that once-daily regimens and reduced pill burden are associated with higher adherence to ART [43,44]. Lower adherence could be a contributory factor to the higher proportion of ART-naive patients on nevirapine who never achieved viral load less than 100 copies/ml (20 vs
$6 \%$ on efavirenz) and worse virological outcomes in ART-experienced children. CHAPAS-3 was not designed to compare the effectiveness of nevirapine and efavirenz, but several other studies in children in resource-limited settings suggest better virological outcomes for the latter [36-38,45-47]. Yet, nevirapine is currently the only NNRTI formulated as all-in-one paediatric FDC. Although developing a similar formulation containing efavirenz could improve treatment adherence and hence virological outcome, this is challenging due to the larger efavirenz dose and higher pharmacokinetic variability due to its pharmacogenetics $[5,48]$.

Adverse events were rare in our study, replicating other paediatric investigations $[17,18,49]$. High nevirapine concentrations were associated with elevated hepatic enzymes in adults, in particular in those with low BMI $[12,50]$, but several other studies including African patients showed a low risk of hepatotoxicity $[10,15,16]$. In CHAPAS-3, although we detected an association between high nevirapine exposures and increased risk of developing more than grade 1 transaminase elevations, all observed events were transient and did not lead to ART substitutions. Likelihood profiling identified a $C_{\min }$ threshold of $12.4 \mathrm{mg} / \mathrm{l}$ as most predictive of these transient events, and although we observed higher transaminase levels during the study when concentrations were above this threshold, they were not significantly different to baseline. Moreover, the baseline values of AST and ALT for ART-experienced children (on nevirapine-based ART for $>2$ years) were not significantly higher than in ART-naive children. Together, these suggest that these findings may have limited clinical relevance. Recent reports hypothesize that nevirapinerelated hepatotoxicity has a genetic cause [51-53]. Similarly, HSRs were rare, possibly due to dose escalation in the first 2 weeks of the study [17]. Small numbers precluded associations with any specific patient characteristics, but they occurred early in the study, before any pharmacokinetics sampling, making it difficult to confirm speculations of their idiosyncratic cause $[4,54]$.

Considering nevirapine's low genetic barrier for viral resistance [8], the risk of nonsuppression decreasing with increasing drug concentrations, and the presented safety profile, maintaining $C_{\min }$ higher than the current target of $3-8 \mathrm{mg} / 1$ could have beneficial effects on general treatment outcomes in African children, and nevirapine concentrations as high as $12.4 \mathrm{mg} / \mathrm{l}$ should not lead to increased risk of adverse events. Results of recent nevirapine population pharmacokinetic analysis in children from CHAPAS-3 [5] show that currently recommended paediatric dosage [41] provides average $C_{\min }$ at the upper range of the $3-8 \mathrm{mg} / 1$ target, even though slow metabolizers determined by CYP2B6 $516 \mathrm{G}>\mathrm{T} \mid 983 \mathrm{~T}>\mathrm{C}$ genotype are at risk of exposures above $12.4 \mathrm{mg} / 1[5]$. 
Our study has several limitations. We could not find a plausible explanation for the detected effect of clinical site on virological outcome, which was not due to small imbalances in other factors as these were either adjusted for or had no association with virological nonsuppression. These centre effects likely reflect residual confounding from factors not captured in our study, either differences in other aspects of management on ART or other local variability in the patient populations, for example, in socio-economic status, distance to clinic, and others. However, we found no evidence that the effect of other independent predictors (adherence, viral load, and nevirapine exposure) varied across centres (i.e. no interaction/heterogeneity) supporting generalizability of these findings to other settings. No genotyping was conducted at enrolment, so we were not able to assess the impact of preexisting NNRTI resistance on response. However, we did not find any evidence of an association between pMTCT (predominantly single-dose nevirapine) and increased risk of nonsuppression, similarly to another recent study [39], suggesting that the impact of preexisting NNRTI resistance may be relatively small compared with the other factors assessed. Most viral loads were matched with nevirapine concentrations measured 12 weeks earlier, and one could argue that drug concentrations measured on the same day as viral load could be more predictive of virological outcome. However, suppression is likely related to maintained drug exposure above a certain threshold, and a random measurement in the time period preceding it could be a better indicator of it. Adherence in our study was only measured in certain time periods, and the same drugtaking pattern was assumed to persist until the next measurement. Most children had only three viral loads after enrolment, and our analysis assumed that no viral rebounds occurred in between. Lastly, our findings should not be generalized to ART based on other drugs, in fact, amongst children enrolled to CHAPAS-3, we found different predictors of virological outcome for children on efavirenz.

\section{Conclusion}

Higher nevirapine concentrations were associated with significantly better virological outcomes, but a meaningful cut-off predictive of increased risk of nonsuppression could not be identified, possibly due to the effects of the combined NRTIs. Lower viral loads at ART initiation and higher treatment adherence were the most predictive determinants of virological suppression. The outcome was further affected by pre-ART $\mathrm{CD}^{+}$cell percentage and clinical site. Adverse events were rare and, even though we detected an association between nevirapine $C_{\min }$ more than $12.4 \mathrm{mg} / 1$ and transaminase elevations, this is of limited clinical relevance due to their transient character. Treatment initiation at lower viral load and higher $\mathrm{CD}^{+}{ }^{+}$cell percentage, increased adherence, and maintaining average $C_{\text {min }}$ higher than current target could have a positive effect on virological suppression of African children treated with nevirapine.

\section{Acknowledgements}

We thank all the children and the staff from all the centres involved in the CHAPAS-3 study. The study was a joint collaboration between the following institutions: JCRC, Kampala, Uganda; Baylor-Uganda, Paediatric Infectious Disease Centre, Mulago Hospital, Uganda; University Teaching Hospital, School of Medicine, Lusaka, Zambia; JCRC, Gulu, Uganda; MRC CTU, London, UK; Radboud University Medical Centre, Nijmegen, the Netherlands; University of Cape Town, Cape Town, South Africa. The Division of Clinical Pharmacology at the University of Cape Town would like to gratefully acknowledge Novartis Pharma for their support of the development of pharmacometrics skills in Africa.

The work was supported by the European Developing Countries Clinical Trials Partnership - EDCTP (IP.2007.33011.006), Medical Research Council (MRC) UK, Department for International Development UK, Ministerio de Sanidad y Consumo, Spain, and the World Health Organization (WHO). Cipla Ltd. donated first-line antiretrovirals. The drug assays were supported in part by the National Institute of Allergy and Infectious Diseases of the National Institutes of Health (UM1 AI068634, UM1 AI068636 and UM1 AI106701, U01 AI068632), the Eunice Kennedy Shriver National Institute of Child Health and Human Development (NICHD), and the National Institute of Mental Health (AI068632). The content is solely the responsibility of the authors and does not necessarily represent the official views of any funders.

Author contributions: Designed and conducted the study (V.M., C.K., A.K., D.M.G., D.B., A.S.W., and H.M.); assayed the samples (L.W.); provided primary data for use in the analyses (A.C.); designed, conducted, and interpreted the analyses (A.B., A.S.W., P.D., and H.M.); drafted the manuscript (A.B. and A.S.W.); critically revised the manuscript and approved its submission (all authors).

\section{Conflicts of interest}

A.B., A.C., V.M., C.K., A.K., A.S.W., D.M.G., H.M., and D.B. received support through grants from EDCTP; A.C., A.K., A.S.W., and D.M.G. additionally received grants from MRC, UK; H.M. additionally declares support in part by the National Research Foundation of South Africa, grant 90729. The remaining authors have no conflicts of interests to disclose. 


\section{References}

1. L'homme RF, Kabamba D, Ewings FM, Mulenga V, Kankasa C, Thomason MJ, et al. Nevirapine, stavudine and lamivudine pharmacokinetics in African children on paediatric fixed-dose combination tablets. AIDS 2008; 22:557-565.

2. World Health Organisation. Consolidated guidelines on the use of antiretroviral drugs for treating and preventing HIV infection. Geneva: World Health Organisation; 2013. Available at: http:// www.who.int/hiv/pub/guidelines/arv2013/en/. [Accessed: 21 October 2013].

3. Wyen $\mathrm{C}$, Hendra $\mathrm{H}$, Vogel $\mathrm{M}$, Hoffmann $\mathrm{C}$, Knechten $\mathrm{H}$, Brockmeyer $\mathrm{NH}$, et al. Impact of CYP2B6 983T > C polymorphism on nonnucleoside reverse transcriptase inhibitor plasma concentrations in HIV- infected patients. I Antimicrob Chemother 2008; 61:914-918.

4. Dickinson L, Chaponda M, Carr DF, van Oosterhout JJ, Kumwenda J, Lalloo DG, et al. Population pharmacokinetic and pharmacogenetic analysis of nevirapine in hypersensitive and tolerant HIV-infected patients from Malawi. Antimicrob Agents Chemother 2014; 58:706-712.

5. Bienczak A, Cook A, Wiesner L, Mulenga V, Kityo C, Kekitiinwa $A$, et al. Effect of diurnal variation, CYP2B6 genotype and age on the pharmacokinetics of nevirapine in African children. J Antimicrob Chemother 2016; 71:dkw388[Epub ahead of print].

6. Veldkamp A, Weverling GJ, Lange JM, Montaner JS, Reiss P Cooper DA, et al. High exposure to nevirapine in plasma is associated with an improved virological response in HIV-1infected individuals. AIDS 2001; 15:1089-1095.

7. de Vries-Sluijs TE, Dieleman JP, Arts D, Huitema AD, Beijnen $J \mathrm{H}$, Schutten $M$, van der Ende ME. Low nevirapine plasma concentrations predict virological failure in an unselected HIV-1-infected population. Clin Pharmacokinet 2003; 42:599-605.

8. de Requena GD, Bonora S, Garazzino S, Sciandra M, D'Avolio $A$, Raiteri $R$, et al. Nevirapine plasma exposure affects both durability of viral suppression and selection of nevirapine primary resistance mutations in a clinical setting. Antimicrob Agents Chemother 2005; 49:3966-3969.

9. Havlir D, Cheeseman SH, McLaughlin M, Murphy R, Erice A, Spector SA, et al. High-dose nevirapine: safety, pharmacokinetics, and antiviral effect in patients with human immunodeficiency virus infection. J Infect Dis 1995; 171:537-545.

10. Dong BJ, Zheng Y, Hughes MD, Frymoyer A, Verotta D, Lizak $\mathrm{P}$ et al. Nevirapine pharmacokinetics and risk of rash and hepatitis among HIV-infected sub-Saharan African women. AIDS 2012; 26:833-841.

11. De Maat MMR, ter Heine R, Mulder JW, Meenhorst PL, Mairuhu AT, van Gorp EC, et al. Incidence and risk factors for nevirapine-associated rash. Eur J Clin Pharmacol 2003; 59:457-462.

12. De Requena DG, Jiménez-Nácher I, Soriano V. Changes in nevirapine plasma concentrations over time and its relationship with liver enzyme elevations. AIDS Res Hum Retroviruses 2005; 21:555-559.

13. La Porte CJL, Back DJ, Blaschke T, Boucher CA, Fletcher CV, Flexner $\mathrm{C}$, et al. Updated guideline to perform therapeutic drug monitoring for antiretroviral agents. Rev Antivir Ther 2006; 3:5-14.

14. Almond LM, Boffito $M$, Hoggard PG, Bonora $S$, Raiteri $R$, Reynolds $\mathrm{HE}$, et al. The relationship between nevirapine plasma concentrations and abnormal liver function tests. AIDS Res Hum Retroviruses 2004; 20:716-722.

15. Kappelhoff BS, van Leth F, Robinson PA, MacGregor TR, Baraldi $E$, Montella $F$, et al. Are adverse events of nevirapine and efavirenz related to plasma concentrations? Antivir Ther 2005; 10:489-498.

16. Chu KM, Boulle AM, Ford N, Goemaere E, Asselman V, Van Cutsem G, et al. Nevirapine-associated early hepatotoxicity: incidence, risk factors, and associated mortality in a primary care art programme in South Africa. PLOS One 2010; 5:2-7.

17. Mulenga V, Cook A, Walker AS, Kabamba D, Chijoka C, Ferrier $A$, et al. Strategies for nevirapine initiation in HIV-infected children taking pediatric fixed-dose combination 'Baby Pills' in Zambia: a randomized controlled trial. Clin Infect Dis 2010; 51:1081-1089.
18. Tukei VJ, Asiimwe A, Maganda A, Atugonza R, Sebuliba I, Bakeera-Kitaka $S$, et al. Safety and tolerability of antiretroviral therapy among HIV-infected children and adolescents in Uganda. I Acquir Immune Defic Syndr 2012; 59:274-280.

19. Mulenga V, Musiime V, Kekitiinwa A, Cook AD, Abongomera G, Kenny J, et al. Abacavir, zidovudine, or stavudine as paediatric tablets for African HIV-infected children (CHAPAS-3): an open-label, parallel-group, randomised controlled trial. Lancet Infect Dis 2016; 16:169-179.

20. World Health Organisation. Antiretroviral therapy for HIV infection in infants and children: towards universal access. Geneva: World Health Organisation; 2010. Available at: http://whqlibdoc.who.int/publications/2010/ 9789241599801_eng.pdf. [Accessed: 21 October 2013].

21. Kirkwood TBL. Geometric means and measures of dispersion. Biometrics 1979; 35:908-909.

22. Andersen APK, Gill RD. Cox's regression model for counting processes: a large sample study. Ann Stat 1982; 10:1100-1120.

23. Klein JP, Moeschberger ML. Statistics for biology and health: survival analysis, techniques for censored and truncated data. New York: Springer-Verlag Inc.; 2003.

24. Therneau T. 'A package for survival analysis in $S$ ', version 2.38.2015, http://CRAN.R-project.org/package=survival [ACcessed: 5 May, 2015].

25. R Core Team. R: a language and environment for statistical computing. Vienna, Austria: R Foundation for Statistical Computing; 2015, http://www.R-project.org/ [Accessed: 05 May, 2015].

26. Royston $P, A$ Atman DG. Regression using fractional polynomials of continuous covariates: parsimonious parametric modelling. Appl Stat 1994; 43:429-467.

27. Bienczak A, Denti P, Cook A, Wiesner L, Mulenga V, Kityo C, et al. Plasma efavirenz exposure, sex, and age predict virological response in HIV-infected African children. JAIDS 2016; 73:161-168[Epub ahead of print].

28. Wade AM, Ades AE. Age-related reference ranges: significance tests for models and confidence intervals for centiles. Stat Med 1994; 13:2359-2367.

29. van Heeswijk RP, Veldkamp AI, Mulder JW, Meenhorst PL, Wit FW, Lange JM, et al. The steady-state pharmacokinetics of nevirapine during once daily and twice daily dosing in HIV1-infected individuals. AIDS 2000; 14:F77-F82.

30. Nikanjam M, Kabamba D, Cressey TR, Burger D, Aweeka FT, Acosta EP, et al. Nevirapine exposure with WHO pediatric weight band dosing: enhanced therapeutic concentrations predicted based on extensive international pharmacokinetic experience. Antimicrob Agents Chemother 2012; 56:5374-5380.

31. Van Leth F, Kappelhoff BS, Johnson D, Losso MH, BoronKaczmarska A, Saag MS, et al. Pharmacokinetic parameters of nevirapine and efavirenz in relation to antiretroviral efficacy. AIDS Res Hum Retroviruses 2006; 22:232-239.

32. Haas DW, Gebretsadik T, Mayo G, Menon UN, Acosta EP, Shintani A, et al. Associations between CYP2B6 polymorphisms and pharmacokinetics after a single dose of nevirapine or efavirenz in African Americans. J Infect Dis 2009; 199:872880.

33. Bouazza N, Tréluyer JM, Msellati $P$, Van de Perre $P$, Diagbouga $S$, Nacro B, et al. A novel pharmacokinetic approach to predict virologic failure in $\mathrm{HIV}$-1-infected paediatric patients. AIDS 2013; 27:761-768.

34. Palumbo P, Wu H, Chadwick E, Ruan P, Luzuriaga K, Rodman I, et al. Virologic response to potent antiretroviral therapy and modeling of HIV dynamics in early pediatric infection. I Infect Dis 2007; 196:23-29.

35. Violari A, Lindsey JC, Hughes MD, Mujuru HA, Barlow-Mosha $L$, Kamthunzi $P$, et al. Nevirapine versus ritonavir-boosted lopinavir for HIV-infected children. N Engl / Med 2012; 366:2380-2389.

36. Jittamala P, Puthanakit T, Chaiinseeard S, Sirisanthana V. Predictors of virologic failure and genotypic resistance mutation patterns in Thai children receiving nonnucleoside reverse transcriptase inhibitor-based antiretroviral therapy. Pediatr Infect Dis J 2009; 28:826-830.

37. Kamya MR, Mayanja-Kizza H, Kambugu A, Bakeera-Kitaka S, Semitala F, Mwebaze-Songa P, et al. Predictors of long-term viral failure among Ugandan children and adults treated with antiretroviral therapy. I Acquir Immune Defic Syndr 2007; 46:187-193. 
38. Emmett SD, Cunningham CK, Mmbaga BT, Kinabo GD, Schimana $W$, Swai $M E$, et al. Predicting virologic failure among HIV-1-infected children receiving antiretroviral therapy in Tanzania: a cross-sectional study. J Acquir Immune Defic Syndr 2010; 54:368-375.

39. Musoke P, Szubert AJ, Musiime V, Nathoo K, Nahirya-Ntege P, Mutasa $K$, et al. Single-dose nevirapine exposure does not affect response to antiretroviral therapy in HIV-infected African children aged below 3 years. AIDS 2015; 29:1623-1632.

40. Lundgren JD, Babiker AG, Gordin F, Emery S, Grund B, Sharma $\mathrm{S}$, et al., INSIGHT START Study Group. Initiation of antiretroviral therapy in early asymptomatic HIV infection. $N$ Eng/ J Med 2015; 373:795-807.

41. World Health Organisation. Consolidated guidelines on the use of antiretroviral drugs for treating and preventing HIV Infection. Geneva: World Health Organisation; 2015.

42. Paterson DL, Swindells S, Mohr J, Brester M, Vergis EN, Squier C, et al. Adherence to protease inhibitor therapy and outcomes in patients with HIV infection. Ann Intern Med 2000; 133:21-30.

43. Parienti JJ, Bangsberg DR, Verdon R, Gardner EM. Better adherence with once daily antiretroviral regimens: a meta analysis. Clin Infect Dis 2009; 48:484-488.

44. Nachega JB, Parienti IJ, Uthman OA, Gross R, Dowdy DW, Sax $\mathrm{PE}$, Gallant JE, et al. Lower pill burden and once-daily antiretroviral treatment regimens for HIV infection: a meta-analysis of randomized controlled trials. Clin Infect Dis 2014; 58:1297-1307.

45. Lowenthal ED, Ellenberg JH, Machine E, Sagdeo A, Boiditswe S, Steenhoff AP, et al. Association between efavirenz-based compared with nevirapine-based antiretroviral regimens and virological failure in HIV-infected children. JAMA 2013; 309:18031809.

46. Duong T, Judd A, Collins IJ, Doerholt K, Lyall H, Foster C, Butler K, et al. Long-term virological outcome in children on antiretroviral therapy in the UK and Ireland. AIDS 2014; 28:2395-2405.
47. Bunupuradah T, Sricharoenchai S, Hansudewechakul R, Klinbuayaem V, Teeraananchai S, Wittawatmongkol O, et al. Risk of first-line antiretroviral therapy failure in HIV-infected Thai children and adolescents. Pediatr Infect Dis / 2015; 34:e58-e62.

48. Bienczak A, Cook A, Wiesner L, Olagunju A, Mulenga V, Kityo $C$, et al. The impact of genetic polymorphisms on the pharmacokinetics of efavirenz in African children. Br J Clin Pharmacol 2016; 82:185-198.

49. Lapphra K, Vanprapar N, Chearskul S, Phongsamart W, Chearskul P, Prasitsuebsai W, Chokephaibulkit K. Efficacy and tolerability of nevirapine- versus efavirenz-containing regimens in HIV-infected Thai children. Int J Infect Dis 2008; 12:e33-8.

50. Sanne I, Mommeja-Marin H, Hinkle J, Bartlett JA, Lederman MM, Maartens G, et al. Severe hepatotoxicity associated with nevirapine use in HIV-infected subjects. I Infect Dis 2005; 191:825-829.

51. Phillips E, Bartlett JA, Sanne I, Lederman MM, Hinkle J, Rousseau $\mathrm{F}$, et al. Associations between HLA-DRB1*0102, HLA$B * 5801$, and hepatotoxicity during initiation of nevirapinecontaining regimens in South Africa. I Acquir Immune Defic Syndr 2013; 62:e55-e57.

52. Ciccacci C, Borgiani P, Ceffa S, Sirianni E, Marazzi MC, Altan AM, et al. Nevirapine-induced hepatotoxicity and pharmacogenetics: a retrospective study in a population from Mozambique. Pharmacogenomics 2010; 11:23-31.

53. Haas DW, Bartlett JA, Andersen JW, Sanne I, Wilkinson GR, Hinkle J, et al. Pharmacogenetics of nevirapine-associated hepatotoxicity: an adult AIDS Clinical Trials Group collaboration. Clin Infect Dis 2006; 43:783-786.

54. Hall DB, Macgregor TR. Case-control exploration of relationships between early rash or liver toxicity and plasma concentrations of nevirapine and primary metabolites. HIV Clin Trials 2007; 8:391-399. 